\title{
Nanoscale
}

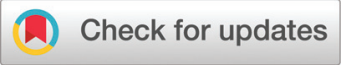

Cite this: Nanoscale, 2017, 9, 9606

\section{Multifunctional electrospun polymeric nanofibrous mats for catalytic reduction, photocatalysis and sensing $\dagger$}

\author{
Osman Arslan (D) * and Tamer Uyar (D) *
}

Received 13th April 2017. Accepted 20th June 2017

DOI: $10.1039 / \mathrm{c} 7 \mathrm{nr} 02658 \mathrm{~g}$ rsc.li/nanoscale

\begin{abstract}
Fabrication and decoration of flexible Nylon 6,6 polymeric nanofibrous mats for production of multifunctional electrospun material was accomplished via visible light-emitting surface-protected silicon quantum dots (Si QD), ZnO nanoparticles ( $\mathrm{ZnO} N \mathrm{~N}$ ) and Pd nanocubes (Pd NC). UV-range light was utilized for Si QD production and, after hydrolysis/condensation together with nucleation and growth reactions, aminemodified, fluorescent Si QD were obtained. Additionally, available molecular groups on the Si QD coated onto the polymeric nanofibrous mats provided further attachment of metal oxide and metal NP for various catalytic purposes. Analytical investigations showed that visible-light emission could be maintained on the Nylon 6,6 mats for trinitrotoluene (TNT) sensing. Also, due to consecutive NP decoration, multifunctional, polymeric, flexible nanofibrous mats were obtained. Experiments revealed that fabricated multifunctional mats could reduce molecules such as paranitrophenol effectively or decompose waste dyes such as methylene blue via photocatalytic experiments, and sense the pollutant TNT in aqueous solutions as an all-in-one concept.
\end{abstract}

\section{Introduction}

Synthesis and efficient decoration of polymeric, flexible nanofibrous mats requires a well-designed strategy. These efficient materials can carry out many functions on an available platform. Therefore, the requisite chemical structures and methods must be engineered very delicately to provide a stepby-step route for enhancing the operative character of these polymeric nanofibrous mats. Previous methods of the silicon (Si) precursor-reducing reactions for Si nanoparticles (NP) and Si quantum dots (QD) required harsh and highly reductive environments. Hence, our previous work, which investigated the formation of Si QD and decoration of Nylon 6,6-nanofiber (Nylon 6,6-NF), simply demonstrated that the mild and lightcontrolled reduction of the alkoxysilane precursor is possible for large-scale fabrication of Si QD. ${ }^{1}$ From this perspective, high temperatures and pressure, extreme conditions, protec-

Institute of Materials Science \& Nanotechnology, UNAM-National Nanotechnology Research Center, Bilkent University, 06800 Ankara, Turkey.

E-mail: uyar@unam.bilkent.edu.tr, arslan@unam.bilkent.edu.tr

$\dagger$ Electronic supplementary information (ESI) available: Further Si QD, ZnO and Pd NC characterization, average diameter analysis for each decoration, visual picture of the multifunctional mat flexibility, sensing, photocatalysis and catalytic reduction details, SEM and EDX analysis after the each application, table of the quantitative detection of the atomic entities and a video for the visual appearance and flexibility of the multifunctional nanofibrous mat. See DOI: $10.1039 / \mathrm{c} 7 \mathrm{nr} 02658 \mathrm{~g}$ tive gas environments and tedious procedures were eliminated for the surface-modified and controlled synthesis of the Si QD. ${ }^{1-3}$ Interestingly, when the (3-aminopropyl)triethoxysilane (APTES) precursor is used, extremely abundant functional groups (e.g., alkoxides, hydroxides, and amine groups) in the aqueous atmosphere seem very suitable environments for progressive chemical organizations such as metal ( $\mathrm{Pd}, \mathrm{Au})$.

$\mathrm{NP}$ attachment via $-\mathrm{NH}_{2}$ groups or metal oxide $\left(\mathrm{TiO}_{2}, \mathrm{ZnO}\right)$ decoration via $-\mathrm{OH}$ or alkoxides because the final nanostructure behaves as a nanocoating with a thickness of only a few nanometera. ${ }^{1}$ As one of the most studied nanomaterial fabrication techniques in recent years, electrospinning has been extensively investigated. It is possible to utilize polymeric or nanocomposite formulations with different mixtures and chemical structures. Electrospinning provides one-dimensional (1D) nanostructures exhibiting high surface area-tovolume and length-to-diameter ratios for varied applications, so these new materials can be used for filtration, optical, sensing, or wound-dressing applications. ${ }^{4-7}$ In the present study, light-controlled production of Si QD was achieved and coupled with metallic (Pd nanocube (Pd NC)) and metal oxide (ZnO NP, ZnO NP) NP to decorate the surface of the electrospun Nylon 6,6-NF by dip coating for development of multifunctional mats. Nylon 6,6-NF was selected as a nanofibrous mat due to its known chemical structure, as well as its easy electrospinnable, polymeric, polar, labile, and non-fluorescent features. Additionally, in its pristine form, nanofibrous mats 
are ready for further bonding for the next step of QD/NP decorations. ${ }^{8-10}$ Nevertheless, it is possible to utilize other polymeric NF providing that they have the required features for the construction of the multifunctional nanofibrous mat. It is known that the emission features of the QD-based nanomaterials are determined by the environmental effects together with surface and functional properties. ${ }^{11,12} \mathrm{Si}$ QDcovered Nylon 6,6-NF emits visible green light due to the dipped Si QD nanocoating. Hence, the additional functionalities of metal (Pd NC) and metal oxide (ZnO NP) NP can be introduced onto the nanofibrous mats via $\mathrm{NH}_{2}-$ and -alkoxy/ hydroxyl features for catalytic reduction and photocatalytic applications in addition to the fluorescent feature. In our previous studies, we have shown that electrospun nanofibrous mats of cellulose acetate, ${ }^{13}$ polycaprolactone, ${ }^{14}$ polysulfone, ${ }^{15}$ and Nylon $6,6^{16}$ can be surface-functionalized with various nanostructures (e.g., gold nanoclusters, $\left.{ }^{13,14} \mathrm{ZnO}^{15,16}\right)$ for applications such as heavy-metal removal, ${ }^{13}$ sensing, ${ }^{14}$ and photocatalysis. ${ }^{15,16}$ It is also possible to surface-functionalize many other electrospun NF of polymers or polymeric composites. ${ }^{4-6}$ For surface-functionalization of materials, sol-gel, ${ }^{17,18}$ graft polymerization, ${ }^{19,20}$ plasma treatments ${ }^{21,22}$ and impregnation techniques ${ }^{23,24}$ can be used.

The most admirable advantages of functional nanofibrous mats are their wide serviceability with all their functionalities to provide high surface area/low volume materials. Therefore, sustainable structures with more productive, efficient, low-cost and low-waste features can be produced. Hence, it is very important to design smart nanomaterials that can do many things simultaneously. Fabricated multifunctional nanofibrous mats can be effectively utilized for water-treatment, filtration, molecular-sensing, and catalytic applications. Additionally, by further design and chemical modifications, these multifunctional nanofibrous materials can be used for various applications depending on the functionality they present. Here, we hierarchically designed and surface-functionalized electrospun Nylon 6,6 NF in step-by-step decoration with Si QD, ZnO NP, and Pd NC to obtain multifunctional nanofibrous mats for 2,4,6-trinitrotoluene (TNT) sensing, photocatalysis and catalytic reduction reactions in an all-inone concept.

\section{Results and discussion}

In the present study, a multifunctional nanofibrous mat, designed by structural and chemical means, was presented. A polymeric electrospun nanofibrous Nylon 6,6-NF mat was used as a template to transform it into a multifunctional nanomaterial by repetitive decoration with $\mathrm{Si} \mathrm{QD}, \mathrm{ZnO} \mathrm{NP}$, and Pd NC. The flexible nature of the Nylon 6,6-NF and controllable chemical structures cooperatively contributed to the functional and operative features of the final material. The full concept of the multifunctional electrospun mat fabrication is highlighted in Fig. 1. As shown, Si QD, ZnO NP, and Pd NC were separately synthesized or prepared, and electrospun Nylon 6,6-NF mats were utilized for multifunctional nanofibrous mat construction by dip coating. Every single step of the nanostructuredecoration process introduced a new inorganic QD or NP functionalization/character into the electrospun, nanofibrous Nylon 6,6 mats. The Si QD solution can carry $-\mathrm{NH}_{2}$ and -OR/$\mathrm{OH}$ groups so, by keeping the latter functionality in the NF, repetitive decoration of inorganic NP was applied. In the decoration process, Si QD (Fig. 2a and b) provided emission of visible light, which allowed the detection of TNT via special intermolecular interactions. In addition, ZnO NP (Fig. 2c and d) decoration provided photocatalytic activity, and Pd NC (Fig. 2e-g) were used to reduce the nitroaromatic paranitrophenol (PNP) catalytically by hydrogenation. All functionalities were compiled in one multifunctional electrospun nanofibrous Nylon 6,6-NF mat (Fig. 1) and they could be used selectively for catalytic and sensing applications.

The decoration sequence has an important role because Pd NC before the ZnO NP caused diminishing of Pd NC activity, as investigations showed. Technically, Si QD decoration by dip coating (Si QD@Nylon 6,6-NF) was always applied prior to ZnO NP and Pd NC. This is because its decoration leads to formation of a fluorescent nanocoating throughout the Nylon 6,6-NF, thereby allowing the groups available on the surface of the Si QD to remain active on the Nylon 6,6-NF surface, which can interact and stabilize the Pd NC by coupling. After Pd NC decoration, there are enough $-\mathrm{NH}_{2}$ groups for TNT sensing by specific molecular interactions.

$\mathrm{Si}$ QD production was performed via reduction of $\mathrm{C}_{6} \mathrm{H}_{17} \mathrm{NO}_{3} \mathrm{Si}$ (APTES) with sodium ascorbate in aqueous solution with UV-light. Scanning transmission electron microscopy (STEM) (Fig. 2a) with size investigation (Fig. S1a†), optical properties under visible and UV light (Fig. 2b), high-resolution X-ray photoelectron spectroscopy (XPS) of Si 2p (Fig. S1b $\dagger$ ), photoluminescence (PL) (Fig. S1c $\dagger$ ) and PL peak deconvolution (Fig. S1d $\dagger$ ) of Si QD were undertaken. Due to the facile nature of the Si QD formation, protected and surface-modified QD were obtained (Fig. 2a). Si QD were spherical without remarkable aggregation, and they could be stored for $75 \mathrm{~h}$ without $\mathrm{SiO}_{2}$ formation and precipitation. Results showed that Si QD to have a crystalline silicon core and a possible silica shell was available to carry $\mathrm{NH}_{2}-$ groups together with alkoxy and hydroxyl parts. Cyan-green emission was observed under UV light (Fig. 2b) and had emission maxima at $\lambda_{\mathrm{em}}=480 \mathrm{~nm}$ (Fig. S1c $\dagger$ ). Observations under different excitation wavelengths revealed that, in the smaller excitation wavelengths, broad and asymmetric PL peak shapes were visible, revealing a multi-asymmetric character (Fig. S1d $\dagger$ ). The PL peak could be fitted into two peaks at $425 \mathrm{~nm}(2.91 \mathrm{eV})$ and another positioned at $515 \mathrm{~nm}(2.50 \mathrm{eV})$. The lower-energy peak possibly originated from surface defects together with surface effects. After the decoration of Si QD, ZnO NP (Fig. 2c and d) were used to decorate the surface of the Si QD-decorated Nylon 6,6-NF mats (ZnONP@SiQD@Nylon 6,6-NF). The transmission electron microscopy (TEM) image (Fig. 2c), high-resolution (HR)-TEM image (Fig. 2d), crystallinity (which was detected by $\mathrm{X}$-ray diffraction (XRD) patterns; Fig. S2a $\dagger$ ) and size-distri- 

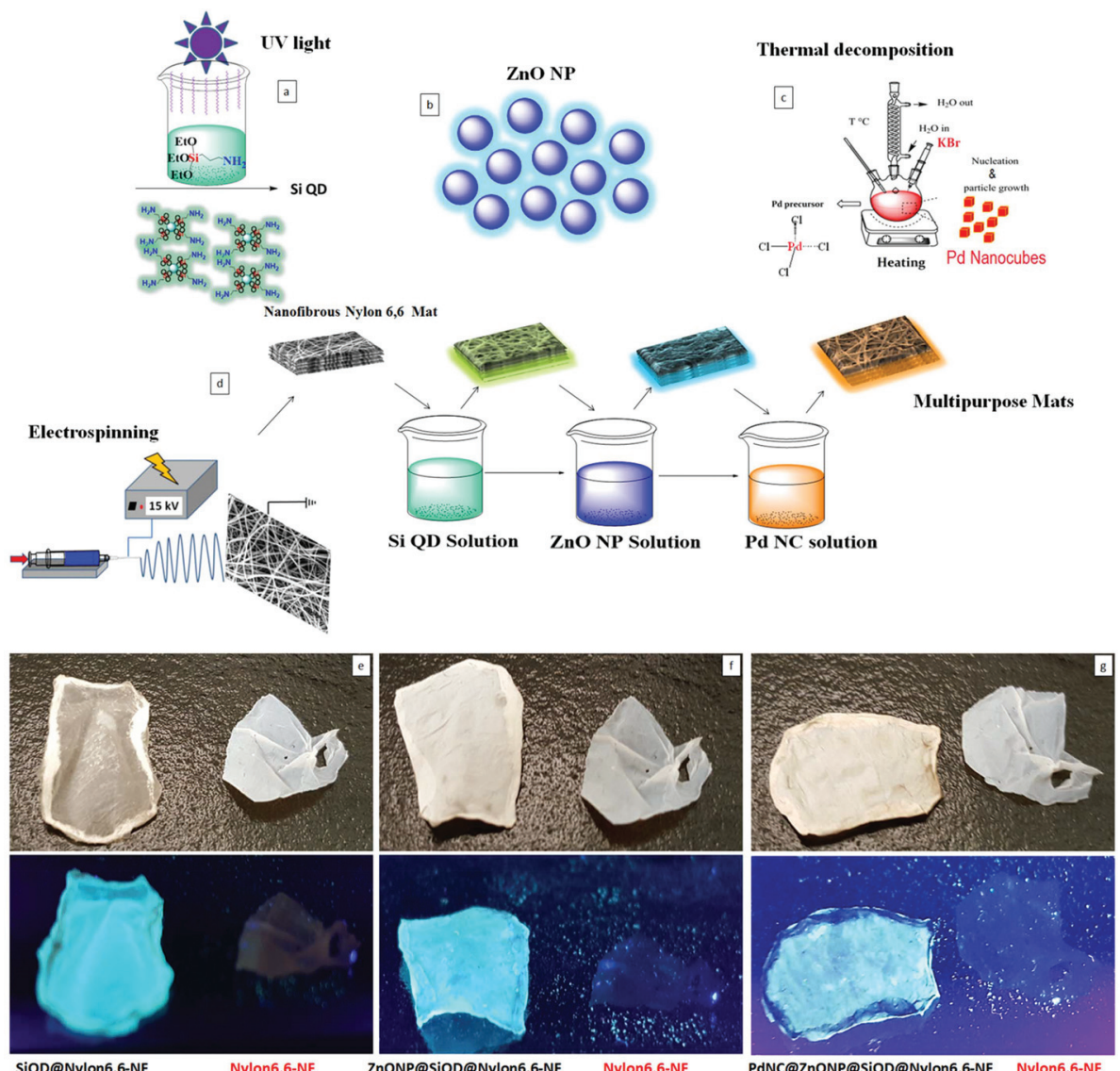

Fig. 1 (a) Si QD synthesis with UV light (b) ZnO NP (c) thermal decomposition method for Pd NC synthesis and (d) electrospinning method for Nylon 6,6-NF and consecutive nanostructure deposition for multifunctional mats. Visual appearances of the particle decorated (black denotation) and pristine (red denotation) Nylon 6,6-NF with and without UV light, respectively (e) SiQD@Nylon 6,6-NF (f) ZnONP@SiQD@Nylon 6,6-NF and (g) PdNCaZnONPaSiQDaNylon 6,6-NF.

bution analysis (Fig. S2b†) for commercially obtained ZnO NP are shown. TEM images were utilized to collect the data for the size-distribution charts by counting $100 \mathrm{NP}$ for the sample and plotting with respect to their frequencies. Enlarged TEM images showed that ZnO NP had a quasi-spherical morphology (Fig. 2d) and mean particle size was $\approx 15 \mathrm{~nm}$ (Fig. S2b $\dagger$ ), which is in agreement with the mean crystal-size values of the NP estimated using Scherrer's equation

$$
D=K \lambda / \beta \cos (\theta)
$$

where $K$ is the wavelength of the X-ray employed, $\beta$ is the fullwidth at half-maximum in radiation of the peak, $K$ is a constant, and $\theta$ is the diffraction angle of the XRD peak. The wellresolved lattice planes with a $d$-spacing of $0.26 \mathrm{~nm}$ for wurtzite (JCPDS 36-1451) ZnO [002] was detected and denoted in the HR-TEM image ${ }^{25,26}$ (Fig. 2d). For Pd NC (Fig. 2e-g) synthesis, a thermal decomposition method was employed at $80^{\circ} \mathrm{C}$. From the literature, it is known that available halogen anions $\left(\mathrm{I}^{-}, \mathrm{Br}^{-}, \mathrm{Cl}^{-}\right)$with surface-directing agents control and govern the nuclei formation, growth, and facet control of monodispersed, anisotropic Pd NP. The utilized polyvinylpyrrolidone (PVP) favored nanocube formation due to "capping" onto the facets of the growing NP, and Pd nanostructures were synthesized with controlled size and facets. Fig. 2e shows the TEM images of the Pd NC size distribution (Fig. S3a†). In Fig. 2f, a welldefined cubic shape and lattice fringe is clearly visible, in which an interatomic distance of $\approx 0.225$ [111] nm is measured as a single Pd NC. The measured mean size was $\approx 13 \mathrm{~nm}$ (Fig. S3a†). Optical absorption measured by UV spectra showed two regions in Fig. S3b, $\uparrow$ as can be anticipated from its visual appearance (Fig. 2g). HR-TEM and TEM investigations showed that nanocubes had corners with slightly curved structures (Fig. 2f) but, nevertheless, single-phase Pd NC formation was apparent. $^{27,28}$

The decoration steps on the Nylon 6,6-NF mats are summarized in Fig. 1. Si QD (Fig. 2a), ZnO NP (Fig. 2c) and Pd NC (Fig. 2e) were dip coated onto the electrospun (Fig. 1d) Nylon 6,6 mats. After each decoration, the visual appearance under visible and UV light are presented. Fig. 1e shows that the mats became slightly pale and highly fluorescent, which helped to maintain their appearance and fluorescent feature (Fig. 1f and g). Pd NC decoration resulted in a slightly yellow product but visible emission could still be seen under UV light (Fig. 1f 


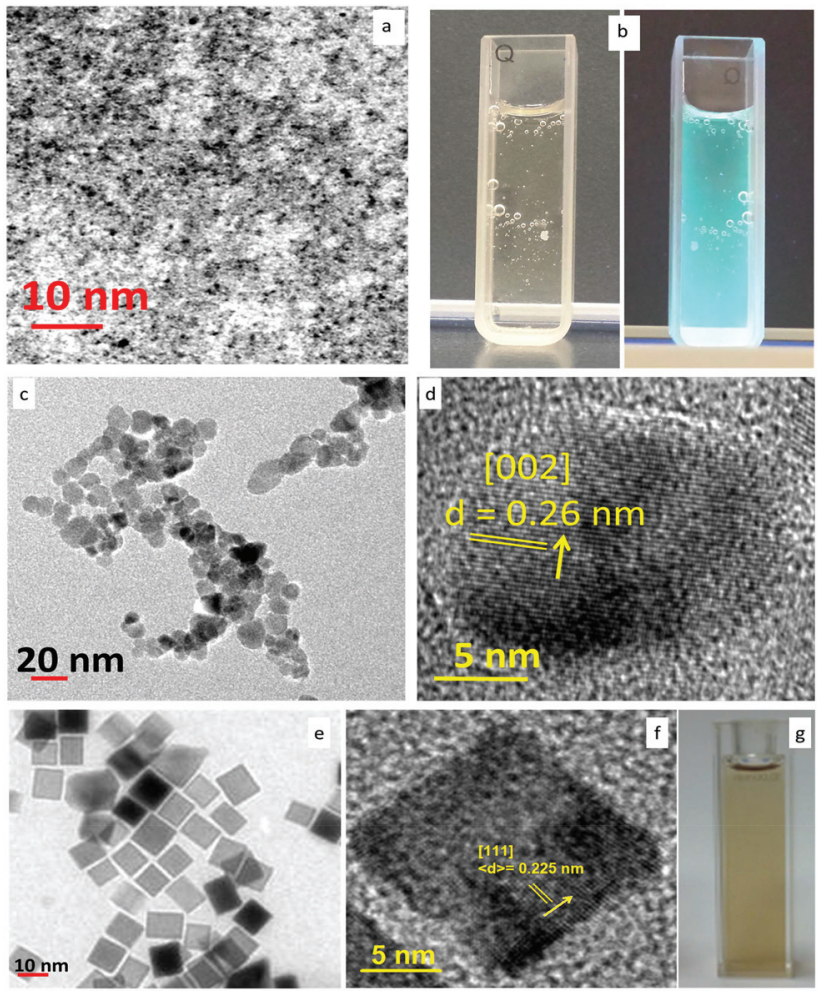

Fig. 2 Representative (a) Si QD STEM image (b) Si QD solution under visible (left) and UV light (right) (c) TEM image of ZnO NP (d) HR-TEM image of $\mathrm{ZnO} N P$, [002] fringe measurement $(d=0.26 \mathrm{~nm})$ (e) TEM image of Pd NC (f) HR-TEM image of single Pd NC, [111] fringe measurement $(d=0.225 \mathrm{~nm})$ and $(\mathrm{g}) \mathrm{Pd} \mathrm{NC}$ solution.
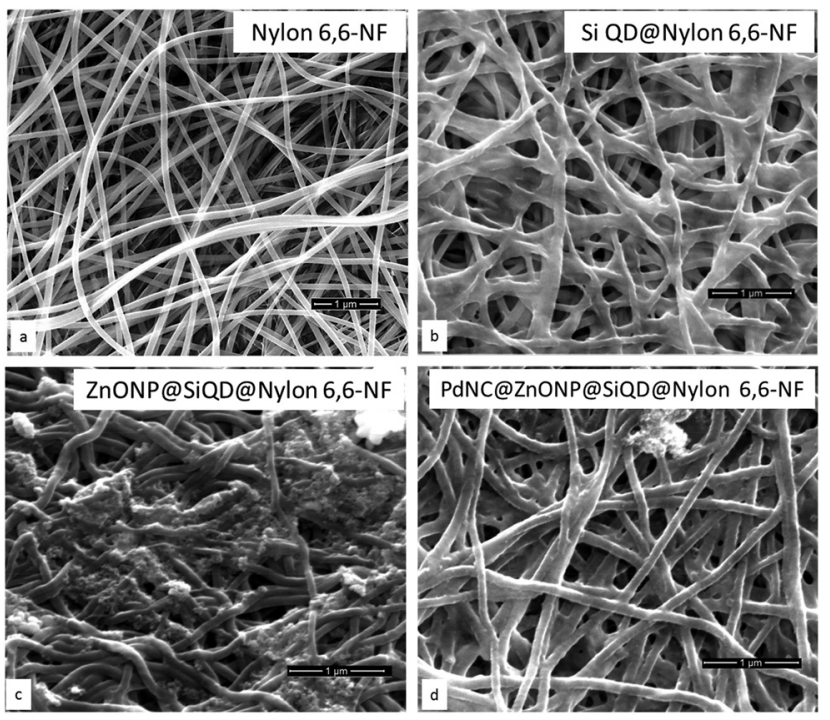

Fig. 3 Representative SEM images of (a) Nylon 6,6-NF (b) SiQD aNylon 6,6-NF (c) ZnONP@SiQDaNylon 6,6-NF and (d) PdNC@ZnONP@SiQD@ Nylon 6,6-NF.

and g). Scanning electron microscopy (SEM) investigation (Fig. 3 and $\mathrm{S} 4 \dagger$ ), energy-dispersive X-ray (EDX) mapping (Fig. 4) and detailed XPS analysis (Fig. 5) for each decoration
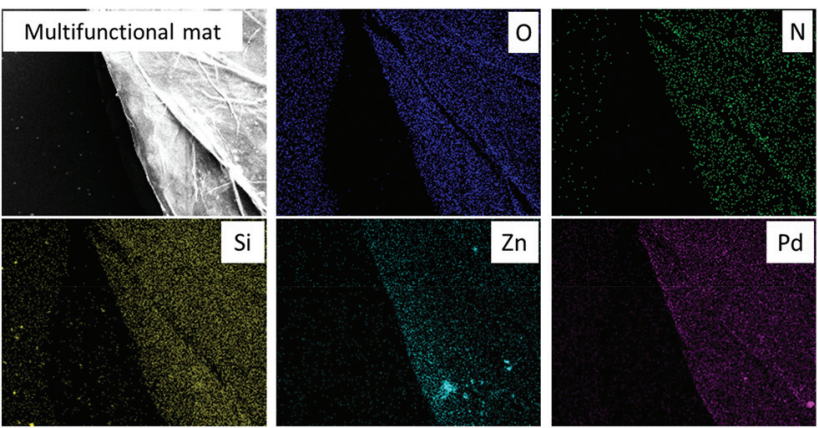

Fig. 4 Representative SEM image and EDX mapping for each atom for PdNC@ZnONP@SiQD@Nylon 6,6-NF except the $C$ atom.

revealed that the Nylon 6,6-NF diameter did not change remarkably upon repetitive decoration, and that Si QD nanocoating was very homogeneously available on the surface of Nylon 6,6-NF. Every nanostructure could be easily detected as seen in the EDX maps except the excluded C map (Fig. 4). Additionally, SEM showed that ZnO NP formed clusters on mats (Fig. 3c), which was due to the surface $-\mathrm{OH}$ groups causing local agglomerations..$^{29,30}$ Even though photocatalytic investigation was quite efficient, $\mathrm{ZnO}$ NP clusters were detectable via EDX mapping and SEM. Surface modification of the electrospun nanofibrous mats provided a wide gallery of the applications that could be used, such as photocatalysis, sensing and catalytic reduction. TEM (Fig. 6a) and STEM (Fig. 6b) investigations of the multifunctional PdNC@ZnONP@SiQD@Nylon 6,6-NF showed that NP were homogeneously distributed on the nanofibrous mat. Fig. $6 \mathrm{c}$ is a representative STEM image of the single polymeric NF showing all the decorated NP. Relatively enlarged TEM images are presented in Fig. 6d-f in which Si QD nanocoating, ZnO $\mathrm{NP}$ and Pd NC nanostructures are also visible. HR-TEM images on a single NF showing $\mathrm{d}-\mathrm{d}$ fringes are presented in Fig. $6 \mathrm{~g}$ for Si QD, Fig. $6 \mathrm{~h}$ for ZnO NP, and Fig. 6i for Pd NC. The obtained bead-free NF were yielded from Nylon 6,6 (10\%, w/v) solutions, and the diameter was found to be $90 \mathrm{~nm}$ after counting 100 different NF and was relatively the same after multi-step decoration (Fig. S4a-d广). Once polymeric Nylon 6,6-NF was produced, the nanofibrous mat was dip coated with Si QD, and then ZnO NP were attached onto Si QD-containing groups.

XPS survey spectra of the as-synthesized Si QD (Fig. 5a) and HR-XPS spectra (Fig. 5b) of $\mathrm{N} 1 \mathrm{~s}$ for as-synthesized Si QD showed that the obtained Si QD were surface-modified to provide additional attachment for other NP. When Si QD were utilized to decorate the polymeric Nylon 6,6-NF until the end, XPS analysis of the PdNC@ZnONP@SiQD@Nylon 6,6-NF (Fig. 5c) showed that all the decorated nanostructures could be seen clearly. We found that $\mathrm{N}$ 1s XPS spectra were also changed for PdNC@ZnONP@SiQD@Nylon 6,6-NF (Fig. 5d). Step-by-step development of the NF decoration could be compared with survey XPS spectra (Fig. 5e), and HR-XPS spectra of the $\mathrm{N}$ 1s (Fig. 5f) from each decoration also supported the surface variations. For the PdNC@ZnONP@SiQD@Nylon 

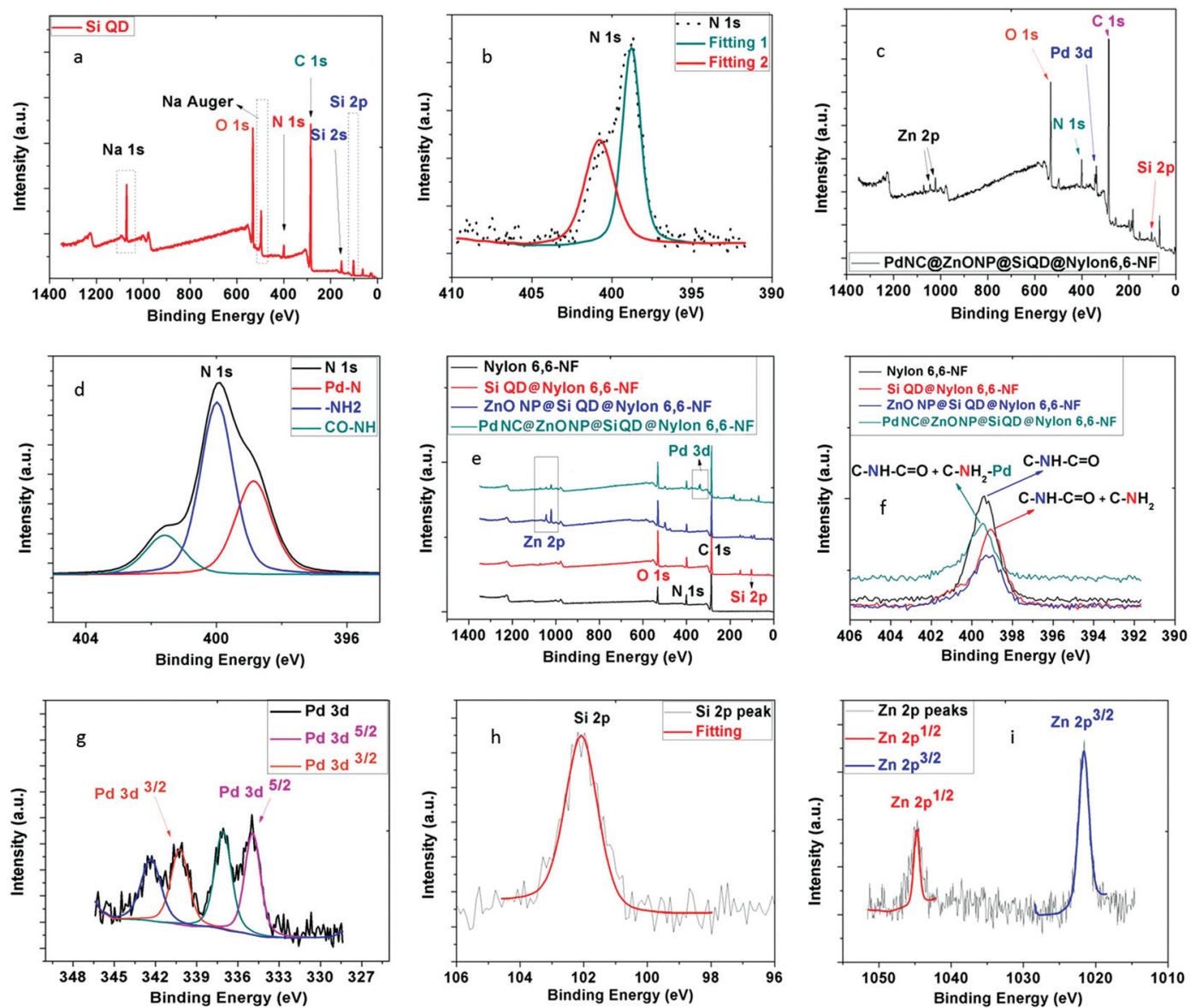

Fig. 5 (a) XPS survey spectra of as-synthesized Si QD (b) HR-XPS spectra of $N$ 1s for as-synthesized Si QD (c) XPS survey spectra of PdNC@ZnONP@SiQD@Nylon 6,6-NF (d) HR-XPS spectra of N 1s for PdNC@ZnONP@SiQD@Nylon 6,6-NF (e) comparison of the XPS survey spectra for consecutive Nylon 6,6-NF decoration ( $f$ ) comparison of the HR-XPS spectra of N 1s consecutive Nylon 6,6-NF decoration (g) HR-XPS spectra of Pd 3d for PdNC@ZnONP@SiQD@Nylon 6,6-NF (h) HR-XPS spectra of Si 2p for PdNC@ZnONP@SiQD@Nylon 6,6-NF (i) HR-XPS spectra of Zn 2p for PdNC@ZnONP@SiQDaNylon 6,6-NF.

6,6-NF, each decorated nanostructure could also be detected with their HR-XPS spectrum for Pd 3d (Fig. 5g), Si 2p (Fig. 5h) and $\mathrm{Zn} \mathrm{2p}$ (Fig. 5i). Detailed XPS analysis of the ZnONP@SiQD@Nylon 6,6-NF (Fig. 5e) survey resulted in detection of a nanocoating via-OH or hydrolysable -OR; Zn 2p and Si 2p electron energy was detected at $100.6 \mathrm{eV}$ for Si 2p, Zn 2p1 at $1044.7 \mathrm{eV}$, and $\mathrm{Zn} \mathrm{2p3}$ at $1022.0 \mathrm{eV}$, respectively. Overall, comparison of the high-resolution $\mathrm{N}$ 1s peaks after each decoration supported the idea of interaction between the $\mathrm{NH}_{2}$ groups visible at $399.5 \mathrm{eV}$ mostly. Peak deconvolution for $\mathrm{N} 1 \mathrm{~s}$ peak showed the last form of the PdNC@ZnONP@ SiQD@Nylon 6,6-NF (Fig. 5f). For pure Nylon 6,6-NF, there was a peak at $399.5 \mathrm{eV}$ that corresponded to the polymeric $\mathrm{N} 1 \mathrm{~s}$, and its deconvolution showed two different and broaden shoulders after Si QD decoration at $399.5 \mathrm{eV}$ and $399.0 \mathrm{eV}$, respectively. The latter peak corresponded to the $-\mathrm{NH}_{2}$ group on the Si QD decorated onto Nylon 6,6-NF. After Si QD nanocoating, ZnO NP decoration did not change the position or intensity of the same $\mathrm{N}$ 1s peak. After the Pd NC decoration, the intensity of the $399.0 \mathrm{eV}$ peak was relatively smaller but the polymeric peak did not change its previous position or intensity. Another peak for the $\mathrm{Pd}-\mathrm{N}$ interaction appeared at $398.6 \mathrm{eV}$ in the deconvolution (Fig. 5d).

After the last decoration (Fig. 7a), a PdNC@ZnONP@ SiQD@Nylon 6,6-NF flexible mat (Fig. S5, Video S1†) was dried at $100{ }^{\circ} \mathrm{C}$ for $10 \mathrm{~min}$ to remove the unreacted alkoxy groups of the Si QD composition. This final PdNC@ZnONP@ SiQD@Nylon 6,6-NF mat was utilized for certain proof-of-principle applications (i.e., photocatalysis of dye, catalytic reduction of PNP, and TNT sensing in water). First, photocatalytic decomposition of an industrial waste, methylene blue (MB) dye, was used as a model pollutant compound. Control experiments were conducted with Nylon 6,6-NF, ZnONP@Nylon 6,6-NF and ZnO@SiQD@Nylon 6,6-NF (Fig. S6 $)$ ). Also, experiments were repeated thrice and the results compared with each other (Fig. S6†) to observe the reproducibility and error limits. The interaction period between the NF and the solution was very short, so MB adsorption (3\%) could be ignored. The UV-Vis spectra (Fig. S6†) and visual images (Fig. 7b) for the initial concentrations of $\mathrm{MB}$ and after $60 \mathrm{~min}$ are presented in Fig. $\mathrm{S} 6 \dagger$ 


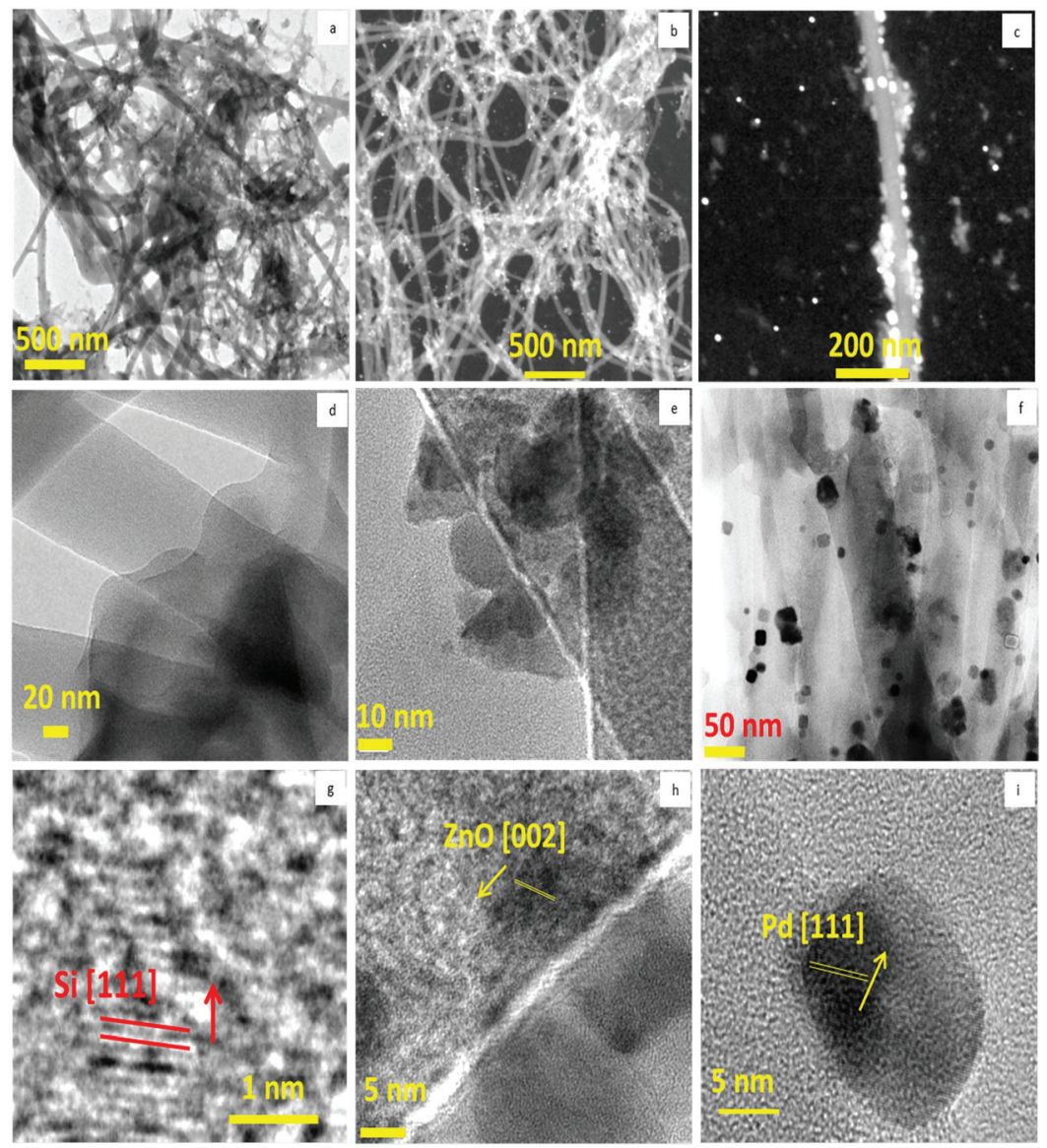

Fig. 6 Representative (a) TEM image of the PdNC@ZnONP@SiQDaNylon 6,6-NF (b) STEM image of the PdNC@ZnONP@SiQD@Nylon 6,6-NF (c) STEM image of a single nanofiber of PdNC@ZnONP@SiQD@Nylon 6,6-NF (d) enlarged TEM image for Si QD decoration for PdNC@ZnONP@SiQD@Nylon 6,6-NF (e) enlarged TEM image for ZnO NP decorated PdNC@ZnONP@SiQD@Nylon 6,6-NF (f) enlarged TEM image for the PdNC@ZnONP@SiQD@Nylon 6,6-NF (g) HR-TEM image for a single Si QD on the PdNC@ZnONP@SiQD@Nylon 6,6-NF with a [111] fringe $(d=0.30 \mathrm{~nm})(\mathrm{h}) \mathrm{HR}-\mathrm{TEM}$ image for a single ZnO NP on the PdNC@ZnONP@SiQD@Nylon 6,6-NF with a [002] fringe ( $d=0.26 \mathrm{~nm})$ (i) HR-TEM image for a single Pd NC on the PdNC@ZnONP@SiQD a Nylon 6,6-NF with a [111] fringe $(d=0.225 \mathrm{~nm})$.

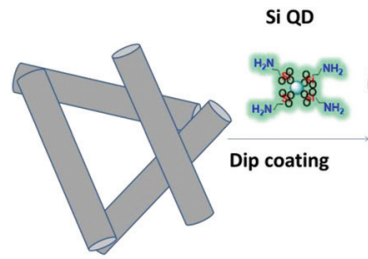

Nylon 6,6-NF

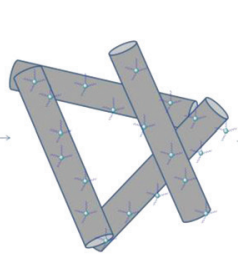

SiQD@Nylon 6,6-NF

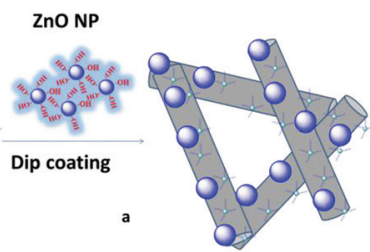

ZnONP@SiQD@Nylon 6,6-NF
Pd NC

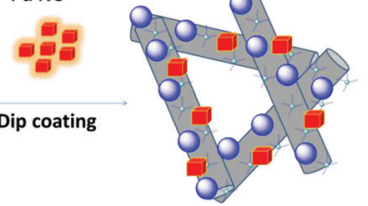

PdNC@ZnONP@SiQD@Nylon 6,6-NF

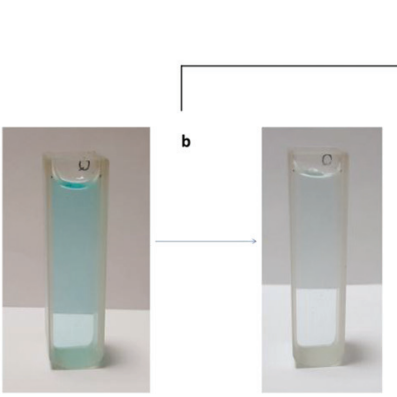

Photocatalysis

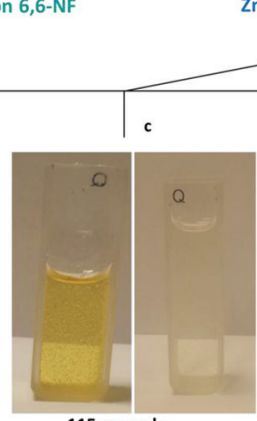

115 seconds
Catalytic Reduction

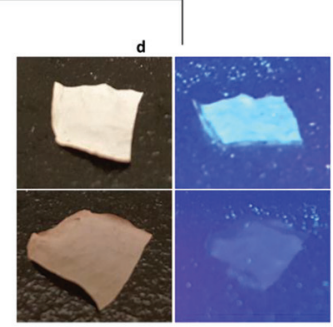

TNT sensing

Fig. 7 (a) Multi-step decoration process of Nylon 6,6-NF (b) photocatalytic degradation of MB by PdNC@ZnONP@SiQD@Nylon 6,6-NF (c) fluorescence TNT sensing by PdNC@ZnONP@SiQD aNylon 6,6-NF (d) representative images of the PNP solution at reduction times of $0 \mathrm{~s}$ and after $115 \mathrm{~s}$. 
for the final mat. Results showed the photocatalytic activity before the Pd NC decoration (ZnONP@SiQD@Nylon 6,6-NF) to be slightly better than that for the multifunctional mat but there was no remarkable variation when compared with PdNC@ZnONP@SiQD@Nylon 6,6-NF. Nylon 6,6-NF showed no $\mathrm{MB}$ decomposition under the same conditions.

Catalytic reduction of PNP was conducted (Fig. 7c and Fig. S7 $\dagger$ ) via fabricated PdNC@ZnONP@SiQD@Nylon 6,6-NF. All catalytic reduction experiments with PdNC@ZnONP@ SiQD@Nylon 6,6-NF were repeated thrice to observe the efficiency of the PdNC@Nylon 6,6-NF and Nylon 6,6-NF, respectively (Fig. $\mathrm{S} 7 \dagger$ ). The reduction process proceeds via $\mathrm{BH}_{4}{ }^{-}$and PNP adsorption onto the Pd NC to form the active intermediate hydride complex to transfer it onto the nitro group, so the surface nature of the Pd NC is extremely important. Adsorption steps follow the Langmuir-Hinshelwood theory, and the rate-determining step for this process consists of the reduction of a nitro compound by surface hydrogenation. ${ }^{31,32}$

PNP reduction was conducted with multifunctional mat and comparison showed that 115 seconds was enough to reduce the $2 \mathrm{mM}$ PNP ( 44 seconds for $1 \mathrm{mM}$ ) while experiment without Pd NC required 36 minutes for the PNP reduction (Fig. S7†).

As another application, TNT detection by PdNC@ZnONP@ SiQD@Nylon 6,6-NF was possible. Previously, we have shown that different concentrations of the TNT solution can be detected (Fig. S8 $\dagger$ ). Although vapor utilization is preferred, TNT is also a carcinogenic organic pollutant found in wastewater. Therefore, we conducted TNT-sensing experiments in aqueous solutions.

Different concentrations of TNT provided quenching which was studied according to the Stern-Volmer equation

$$
I_{0} / I=1+\mathrm{KS}_{\mathrm{V}}[\mathrm{A}]
$$

where $I_{0}$ is the initial fluorescence intensity without analyte, $I$ is the fluorescence intensities of the analyte responses at different concentrations, $\mathrm{KS}_{\mathrm{V}}$ is the quenching constant and [A] is the analyte concentration. Detection based on the SternVolmer equation is shown together with molecular formation of the Meisenheimer complex (Fig. S8†). Different concentrations were analyzed and plotted as in the Volmer-Stern plot. When the multifunctional mat was utilized, the nanofibrous mat changed from white to pink-rose upon exposure to $10 \mathrm{nM}$ TNT solution (Fig. S8†). Visual examination showed that the PdNC@ZnONP@SiQD@Nylon 6,6-NF mat lost its fluorescent character after treatment with aqueous TNT solution. Visual observation and data are also presented in Fig. S8. $\dagger$ For clarity, the error bars for the three lowest concentrations $\left(10 \times 10^{-9} \mathrm{M}\right.$, $1 \times 10^{-7} \mathrm{M}$ and $1 \times 10^{-6} \mathrm{M}$ ) of TNT solutions are presented (Fig. S8b $\dagger$ ).

SEM and EDX atomic mapping investigations (Fig. S9-S11†) were conducted after each application to show that decorated nanostructures remained available on the multifunctional mat. Also, EDX and XPS to compare the quantitative amounts of each deposition showed that the same observations could be made (Table S1†). The amount of active NP is much lower than that of multifunctional nanofibrous mats, so considerably less activity was observed with single NP (PdNC@Nylon 6,6-NF, ZnONP@Nylon 6,6-NF)-decorated mats (Fig. S6 and $\mathrm{S} 7 \dagger$ ). Well engineered, hierarchical, step-by-step deposition of nanostructures onto Nylon 6,6-NF provided an efficient amount of Si QD, ZnO NP and Pd NC on the nanofibrous mats. When compared with the single NP-decorated NF (PdNC@Nylon 6,6-NF or ZnONP@Nylon 6,6-NF), the photocatalytic activity of PdNC@ZnO@SiQD@Nylon 6,6-NF for MB or PNP catalytic reduction by PdNC@ZnO@SiQD@Nylon 6,6-NF showed noticeably high performances. In the absence of Si QD decoration, ZnO NP and Pd NC cannot be effectively decorated, so single NP-decorated NF (PdNC@Nylon 6,6-NF or ZnONP@Nylon 6,6-NF) showed very poor performance due to the minimal amount of functional NP (ZnO NP and Pd NC). Conversely, multifunctional PdNC@ZnONP@SiQD@Nylon 6,6-NF mats showed much better performance because the prior Si QD decoration provided effectively decorated ZnO NP and Pd NC on the Nylon 6,6 NF. In addition, the conducted experiments did not change the fibrous morphology of the electrospun multifunctional mats, as revealed by SEM analyses (Fig. S9-11†).

\section{Materials}

APTES was donated from Evonik Industries. Nylon 6,6 pellets (relative viscosity: $230000-280000)$, formic acid (98-100\%) and sodium ascorbate (99\%) were purchased from SigmaAldrich. All chemicals were used as received without further purification. ZnO NP were purchased from IBU-Tech. Sodium tetrachloropalladate(II) hydrate $\left(\mathrm{Na}_{2} \mathrm{PdCl}_{4} \cdot x \mathrm{H}_{2} \mathrm{O}, 99.95 \%\right.$; Alfa Aesar), PVP ( $M_{\mathrm{w}}: 1300$ 000, Sigma-Aldrich) potassium bromide (KBr, 99\%, Sigma-Aldrich) and ascorbic acid (AA, 99\%, Merck) were used for the synthesis of Pd NC.

\section{Synthesis of surface-protected Si QD and TNT sensing}

For the synthesis of surface-protected Si QD, APTES was dissolved in water and a reducing agent, sodium ascorbate (99\%, Sigma-Aldrich), was introduced at $1: 4$ proportion. The mixture was stirred for $3 \mathrm{~min}$ at $500 \mathrm{rpm}$ and placed under UV light $(300 \mathrm{~W}$, radiated power $=315-400 \mathrm{~nm}(\mathrm{UVA})$ at $13.6 \mathrm{~W}$, and 280-315 $\mathrm{nm}$ (UVB) at $3.0 \mathrm{~W}, 15 \mathrm{~cm}$ length, Ultravitalux, Osram) under constant stirring. After $90 \mathrm{~min}$ of UV treatment, the solution became pale, red-wine, and its fluorescence feature was detected immediately. For sensing experiments, TNT $\left(10 \times 10^{-9} \mathrm{M}\right.$ to $\left.1 \times 10^{-3} \mathrm{M}\right)$ was dropped into the Si QD solution and quenching of fluorescence intensity was measured.

\section{Synthesis of Pd NC}

For the synthesis of Pd NP, a mixture of $0.21 \mathrm{~g}$ of PVP, $0.30 \mathrm{~g}$ of $\mathrm{KBr}$ and $\mathrm{AA}$ was prepared in $16 \mathrm{~mL}$ of water. The solution was stirred for $\approx 30 \mathrm{~min}$ and then $0.114 \mathrm{~g}$ of $\mathrm{Na}_{2} \mathrm{PdCl}_{4} \cdot x \mathrm{H}_{2} \mathrm{O}(x \approx 3)$ in $5 \mathrm{~mL}$ of $\mathrm{H}_{2} \mathrm{O}$ was added. A thermal decomposition reaction was conducted at $80^{\circ} \mathrm{C}$ for $3 \mathrm{~h}$. The available liquid part was 
removed by centrifugation at $1100 \mathrm{rpm}$ for $15 \mathrm{~min}$. Water was removed by a rotavapor and repeat mixtures prepared with toluene to remove the water completely. NP were analyzed with different techniques and redispersed in water for deposition onto the nanofibrous mat.

\section{ZnO NP}

Commercially available (Zincox, IBU-Tech) ZnO NP were utilized for decoration. Size, crystallinity, surface properties and optical features were detected before decoration. Then, $10 \mathrm{mg}$ in $10 \mathrm{~mL}$ aqueous solutions were prepared for decoration.

\section{Electrospinning of Nylon 6,6 NF}

For the electrospinning of polymeric NF, Nylon 6,6 solution was prepared by dissolving $10 \%(\mathrm{w} / \mathrm{v})$ Nylon 6,6 pellets in formic acid and stirring for $3 \mathrm{~h}$ at room temperature. The prepared solution was homogenized by stirring. This was followed by loading into $3 \mathrm{~mL}$ syringes (metallic needle with different inner diameters) and positioned horizontally on the syringe pump (model KDS 101, KD Scientific). The electrode of the high-voltage power supply (AU Series, Matsusada Precision) was clamped onto the metal needle tip and the target aluminum collector was grounded. In general, electrospinning of the polymer solution was performed at an applied voltage of $15 \mathrm{kV}$, tip-to-collector distance of $15 \mathrm{~cm}$ and a solution flow rate of $0.5 \mathrm{~mL} \mathrm{~h}^{-1}$. Nylon 6,6-NF were deposited on a grounded stationary metal collector covered by a piece of aluminum foil. The electrospinning apparatus was enclosed in a Plexiglas ${ }^{\mathrm{TM}}$ box and electrospinning carried out at $25{ }^{\circ} \mathrm{C}$ at a relative humidity of $30 \%$. The electrospun Nylon 6,6 NF were collected onto the aluminum foil and then peeled off as a freestanding mat.

\section{Deposition of Si QD, ZnO NP and Pd NC onto Nylon 6,6 mats}

For decoration onto the Nylon 6,6 nanofibrous mats, a homemade dip coating setup was installed and the nanofibrous mat $(2 \times 2 \mathrm{~cm})$ was removed from the Si QD solution at a withdrawal speed of $100 \mathrm{~mm} \mathrm{~min}^{-1}$. The same procedure was applied for the ZnO NP and Pd NC deposition consecutively after 5 min of drying at $100{ }^{\circ} \mathrm{C}$ after the previous deposition. Morphological, structural, optical and compositional experiments were conducted together with material-application experiments for these decorated nanofibrous mats.

\section{Photocatalytic decomposition}

$\mathrm{MB}\left(\mathrm{C}_{16} \mathrm{H}_{18} \mathrm{~N}_{3} \mathrm{ClS}\right)$ was purchased from Sigma-Aldrich. A stock solution of $\mathrm{MB}\left(1.28 \times 10^{-5} \mathrm{M}\right)$ was prepared and the concentration of $\mathrm{MB}$ in each aqueous solution was measured on an UV-vis spectrophotometer at an absorption at $660 \mathrm{~nm}$.

\section{Catalytic reduction of PNP}

For PNP-reduction experiments, $2 \mathrm{mM}$ of PNP solution was prepared as a stock solution with $\mathrm{H}_{2} \mathrm{O}$ containing $5 \%$ EtOH. From this stock solution, required solutions were taken and diluted into the necessary concentrations. PdNC@ZnONP@ SiQD@Nylon 6,6-NF webs with $1 \times 1 \mathrm{~cm}$ sizes were immersed into $4 \mathrm{~mL}$ of $\mathrm{PNP}$ and $3 \mathrm{~mL}$ of a freshly prepared $\mathrm{NaBH}_{4}$ mixture and stirred in constant speed (100 rpm). Aliquots of the $\mathrm{PNP}-\mathrm{NaBH}_{4}$ solution were taken to observe the reaction process at room temperature until the whole component was reduced. Blank experiments were conducted without any webs. The efficiency of the reduction process was determined using a UV-vis spectrophotometer.

\section{Characterization}

XRD (X'Pert powder diffractometer, PANalytical) patterns were obtained using $\mathrm{Cu} \mathrm{K \alpha}$ radiation at a $2 \theta$ range of 5-80. Investigation of elemental composition (EDX mapping) of the nanofibrous mats before and after decoration was performed together with morphology and diameter analyses with a scanning electron microscope (Quanta 200 FEG, FEI). To avoid the electron-charging effect, nanofibrous mats were coated with $5 \mathrm{~nm}$ of Au (with PECS-682) prior to SEM imaging. The mean diameters of the nanofibrous mats were calculated by counting and measuring 50-100 fiber diameters and plotting them with respect to their frequencies. Non-linear fitting on the obtained fiber diameters gave a mean value with a calculated statistical standard deviation. The surface and elemental composition of NP, nanofibrous mat and decorated mats were performed by XPS (Thermo Scientific). XPS spectra were obtained by a flood gun charge neutralizer system equipped with a monochromated Al K $\alpha$ X-ray source $(h \nu=1486.6 \mathrm{eV})$ from $400 \mathrm{~mm}$ spot size on the nanofibrous mat. Wide energy survey scans were recorded between a binding energy range of 0 and $1360 \mathrm{eV}$ at detector pass energy of $200 \mathrm{eV}$ and with an energy step size of $1 \mathrm{eV}$. High-resolution spectra were obtained at pass energy of $50 \mathrm{eV}$ and with energy steps of $0.1 \mathrm{eV}$ for each atom. Additional size, structure, morphological and surface examinations of the NP and nanofibrous mats were conducted by TEM using a Tecnai G2 F30 system equipped with an EDS detector. Detection of the UV-Vis absorption and PL properties of NP were conducted by UV-Vis spectrophotometers (Cary 100 and Varian 100).

\section{Conclusions}

Multifunctional nanofibrous electrospun Nylon 6,6 mats were fabricated by co-decoration of functional NP (i.e., Si QD, ZnO NP and Pd NC) by dip coating. Si QD nanocoating provides $-\mathrm{OH}$, ethoxy and $-\mathrm{NH}_{2}$ functional groups on the nanofibrous mat, so further NP decoration on this SiQD@Nylon 6,6-NF could be facilitated. From this perspective, after Si QD nanocoating on the Nylon 6,6 mat, ZnO NP and Pd NC were decorated consecutively to fabricate a multifunctional mat: PdNC@ZnONP@SiQD@Nylon 6,6-NF. This multifunctional nanofibrous mat can be used for TNT sensing, PNP reduction and photocatalytic MB decomposition selectively and separately. Analytical investigations showed that the sequence of Pd NC decoration should be done last because its surface should be clean for molecular attachment during hydride complex transfer. The surface multifunctionality, nanofibrous mor- 
phology, effective catalytic and sensing capability, and readily usable features make PdNC@ZnONP@SiQD@Nylon 6,6-NF a novel multifunctional nanomaterial with an all-in-one concept.

\section{Acknowledgements}

O. A. acknowledges the Scientific \& Technological Research Council of Turkey (TUBITAK), TUBITAK BIDEB Fellowships for 2232 programme (project no. 115C095) and financial support. T. U. acknowledges partial support from the Turkish Academy of Sciences - Outstanding Young Scientists Award Program (TUBA-GEBIP). The authors thank Dr Fuat Topuz for Pd NC synthesis and Mustafa Güler for TEM-STEM imaging.

\section{Notes and references}

1 O. Arslan, Z. Aytac and T. Uyar, J. Mater. Chem. C, 2017, 5, 1816-1825.

2 G. Ledoux, J. Gong, F. Huisken, O. Guillois and C. Reynaud, Appl. Phys. Lett., 2002, 80, 4834.

3 W. Sun, C. Qian, K. K. Chen and G. A. Ozin, ChemNanoMat, 2016, 2, 847 .

4 T. Uyar and E. Kny, Electrospun Materials for Tissue Engineering and Biomedical Applications: Research, Design and Commercialization, Woodhead Publishing, 2017, ISBN: 9780081010228.

5 J. H. Wendorff, S. Agarwal and A. Grenier, Electrospinning: materials, processing, and applications, John Wiley \& Sons, 2012.

6 C. Zhang and S. Yu, Chem. Soc. Rev., 2014, 43, 4423-4448.

7 O. Arslan, F. Topuz, H. Erdem, N. Biyikli and T. Uyar, New J. Chem., 2017, 41, 4145-4156.

8 X. Guan, G. Zheng, K. Dai, C. Liu, X. Yan, C. Shen and Z. Guo, ACS Appl. Mater. Interfaces, 2016, 8, 14150-14159.

9 J. Lee, D. Kim, M. G. Mali, S. S. Al-Deyab, M. T. Swihart and S. S. Yoon, Nanoscale, 2015, 7, 19027-19035.

10 N. Wang, Y. Yang, S. S. Al-Deyab, M. El-Newehy, J. Yu and B. Ding, J. Mater. Chem. A, 2015, 3, 23946-23954.

11 H. Althues, J. Henle and S. Kaskel, Chem. Soc. Rev., 2007, 36, 1454-1465.

12 L. E. Alexander and J. N. David, Nat. Nanotechnol., 2016, 11, 661-671.
13 A. Senthamizhan, B. Balusamy, A. Celebioglu and T. Uyar, J. Mater. Chem. A, 2016, 4, 2484.

14 A. Senthamizhan, A. Celebioglu and T. Uyar, Sci. Rep., 2015, 5, 10403.

15 F. Kayaci, S. Vempati, I. Donmez, N. Biyikli and T. Uyar, Nanoscale, 2014, 6, 10224.

16 F. Kayaci, C. Ozgit-Akgun, N. Biyikli and T. Uyar, RSC Adv., 2013, 3, 6817.

17 O. Arslan, E. Arpaç, F. Sayılkan and H. Sayılkan, J. Mater. Sci., 2007, 42, 2138.

18 A. E. Danks, S. R. Hall and Z. Schnepp, Mater. Horiz., 2016, 3, 91.

19 A. Bhattacharyaa and B. N. Misra, Prog. Polym. Sci., 2004, 29, 767.

20 O. Arslan, E. Arpac and H. Sayılkan, J. Inorg. Organomet. Polym. Mater., 2010, 20, 284.

21 P. K. Chua, J. Y. Chena, L. P. Wanga and N. Huang, Mater. Sci. Eng., R, 2002, 36, 143.

22 S. Ben Said, F. Arefi-Khonsari and J. Pulpytel, Plasma Processes Polym., 2016, 13, 1025.

23 L. Delannoy, N. El Hassan, A. Musi, N. N. Le To, J. M. Krafft and C. Louis, J. Phys. Chem. B, 2006, 16, 22471.

24 H. Choi, S. R. Al-Abed, S. Agarwal and D. D. Dionysiou, Chem. Mater., 2008, 20, 3649.

25 O. Arslan, A. P. Singh, L. Belkoura and S. Mathur, J. Mater. Res., 2013, 14, 1947.

26 O. Arslan, L. Belkoura and S. Mathur, J. Mater. Chem. C, 2015, 3, 11965.

27 B. Lim, M. Jiang, J. Tao, P. H. C. Camargo, Y. Zhu and Y. Xia, Adv. Funct. Mater., 2009, 19, 189.

28 M. Liu, Y. Zheng, L. Zhang, L. Guo and Y. Xia, J. Am. Chem. Soc., 2013, 135, 11752-11755.

29 B. Tina, L. Xiao, O. Arslan, H. Cordula, M. Xenia, K. Grieder, B. Wampfler, S. Mathur, P. Wick and H. Krug, Nanotoxicology, 2013, 7, 402-416.

30 S. Tuomela, R. Autio, T. Buerki-Thurnherr, O. Arslan, A. Kunzmann, B. Andersson-Willman, P. Wick, S. Mathur, A. Scheynius, H. F. Krug, B. Fadeel and R. Lahesmaa, PLoS One, 2013, 8, e68415.

31 H. K. Kadam and S. G. Tilve, $R S C A d v ., 2015$, 5, 8339183407.

32 J. Sun, Y. Fu, G. He, X. Sun and X. Wang, Catal. Sci. Technol., 2014, 4, 1742-1748. 